\title{
Characteristics of the spring sand and dust weather and its impact on air pollution in Lanzhou*
}

\author{
$\dagger$ Min-Jin Ma and An-Bao Zhu \\ College of Atmospheric Sciences, Lanzhou University \\ Lanzhou, 730000, China \\ E-mail: minjinma@lzu.edu.cn \\ Fan Ding \\ College of Computer and Communication, Lanzhou University of Technology \\ Lanzhou, 730050, China

\begin{abstract}
Influence of sand and dust weather on air pollution in Lanzhou is discussed by using the particulate matter monitoring data and the ground conventional meteorological observation data during 2001 to 2010. The results are shown as: (1)Sand and dust weather in Lanzhou mainly happens in spring. It presents a 4 years periodicity between the two peaks of occurrence of sand and dust weather. The occurrence is obviously decreased after the peak. (2)During sand and dust weather, air pollution index as well as $\mathrm{PM}_{10}$ concentration rises sharply. The average ratio is 1.16 between $\mathrm{PM}_{10}$ average concentration and $\mathrm{PM}_{10}$ average concentration eliminated sand and dust weather. The occurrence of the sand and dust weather has significantly heightened $\mathrm{PM}_{10}$ average concentration in the spring. The heightening effect becomes obvious with the more occurrence of the sand and dust weather. (3) Variable pressure in 3 hours is positive during the sand and dust weather. The wind direction mainly locates in the range from the north to the east. Visibility is also influenced if the sand and dust weather occurs.
\end{abstract}

Keywords: Sand and Dust Weather; Air Pollution; Lanzhou.

\section{Introduction}

Sand and dust weather as a disastrous weather has obvious spatial-temporal characteristics[1]. Four desert areas in the word are located at North America, Oceania, Central Asia and Middle East. The sand and dust weather is frequently observed in North China during the spring[2]. Especially, due to the special geographical environment, the sand and dust weather frequently occurs in Northwest China. Lanzhou is an important western industrial city in Northwest

\footnotetext{
* This work is supported by the Fundamental Research Funds for the Central Universities (Grant No. lzujbky-2016-17), Drought Meteorological Science Research Fund Project (Grant No. IAM201209) and the Young Scientists Fund of the National Natural Science Foundation of China (Grant No. 41205008).
} 
China. It locates in the loess plateau. Except for anthropogenic emission sources, the sand and dust weather also plays an important role in modulating ambience of air pollution in Lanzhou. Researches show that there is a positive correlation between the annual days of sand-dust storms occurred over the Hexi district in Gansu and the concentration of total suspended particulates in the air in Lanzhou[3]. Sand and dust weather has an evident effect on the concentration of inhaled particulates by using the monitoring data from Auto-Monitoring station[4]. A case study shows that the sandy weather causes serious air pollution accident and make the air quality worse by 2 to 5 time than the normal[5]. Sand and dust weather has an important impact on air quality of Lanzhou and then directly affects the public life and health. In the paper, characteristics of sand and dust weather and contribution to the air pollution in Lanzhou are analyzed by using the air pollution monitoring data and ground meteorological observational data in the recent 10 years.

\section{Materials and Methods}

Data used in the study are the concentration of $\mathrm{PM}_{10}$ monitored by the environmental monitor station and the meteorological observations (including wind speed and direction, pressure, visibility and weather phenomena) in Lanzhou from 2001 to 2010. Statistical methods are used to analyze the impact of sand-dust weather on the air pollution in Lanzhou.

\section{Characteristics of Sand-Dust Weather Variation in Recent 10 Years}

The dynamic factors of sand weather occurred in North China are wind and temperature, and the main resistant factors are vegetation coverage and precipitation[6]. Spring provides benefit factors to form sand and dust weather in Northwest China where Lanzhou is mainly affected. Variation of sand-dust weather occurrence is presented in Figure 1 (Data from January to May in 2001 missed). Lanzhou suffered several sand-dust weather in different intensity each year. Such as in 2010, 15 days sand-dust weather occurs in the spring. The number accounts for $100 \%$ of the total days throughout the year. Meanwhile, in 2004 , the sand-dust weather occurred in the spring accounted for $66.6 \%$ of the year, which reaches the lowest proportion in the spring. The peak days of sanddust weather are in 2002, 2006 and 2010, separately 12, 12, 15 days. The time interval between each peak appears as 4 years, thus it can be seen that the occurrence of sand and dust weather shows a certain periodicity in Lanzhou. 


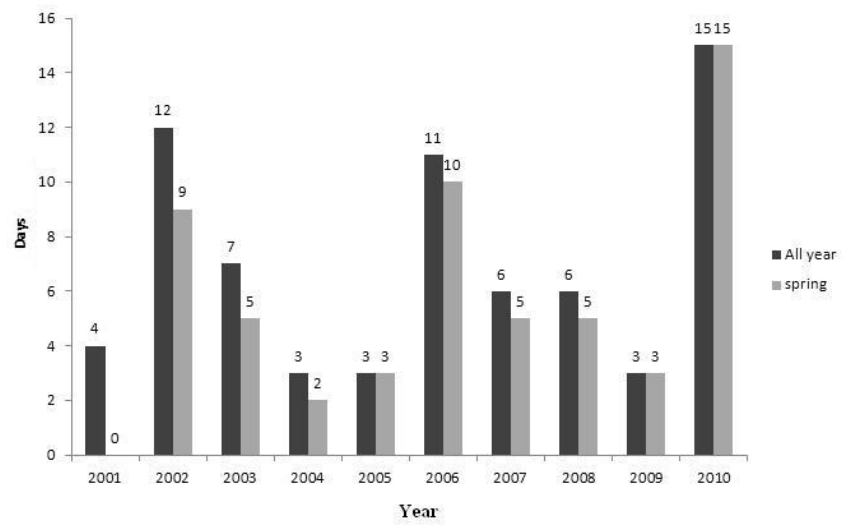

Fig. 1. Occurrence variation of the sand and dust weather of the all year and in the spring.

\section{Influence of Sand and Dust Weather on Air Pollution}

In addition to the man-made emissions, sand and dust weather has also contributed to the air pollution in Lanzhou. As Figure 2, the occurrence of sand and dust weather is consistent with severe air pollution in Lanzhou. Through the correlation analysis, the correlation coefficient of them days is 0.683 (through the correlation test of significant level equal 0.05 ). Thus sandy or dusty weather is an important cause to the serious air pollution in Lanzhou.

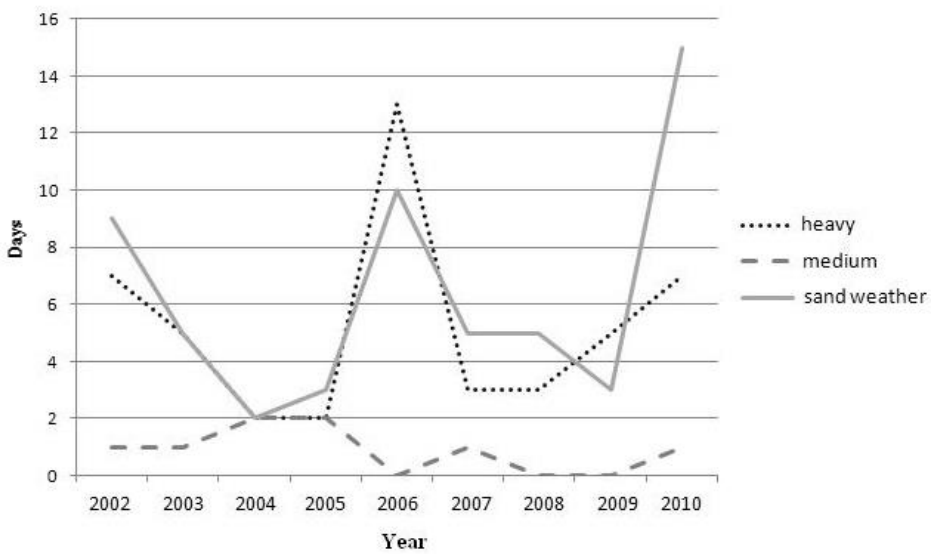

Fig. 2. Variation of the air pollution frequency and the sand and dust weather (in the figure, heavy: heavy air pollution, medium: medium air pollution, sand weather: sand and dust weather).

In order to study the contribution of dust events to air pollution in Lanzhou, the $\mathrm{PM}_{10}$ concentration of air pollution in the spring in recent 10 years is analyzed. Based on the observed weather phenomena, the air pollution in Lanzhou is divided into two kinds of states that are no sand and dust weather and the usual weather. The results show that the average daily $\mathrm{PM}_{10}$ 
concentration is 1.16 times than the average $\mathrm{PM}_{10}$ concentration which days eliminates sand and dust weather (Figure 3). Namely the sand and dust weather contributes a lot to the average $\mathrm{PM}_{10}$ concentration. In the past 10 years, the highest ratio of the average $\mathrm{PM}_{10}$ concentration to the $\mathrm{PM}_{10}$ concentration eliminated sand-dust weather in the spring is 1.29 in 2002. It shows that sand and dust weather has the most significant effect on the average daily $\mathrm{PM}_{10}$ concentration in the spring. However, the ratio is 1.05 in 2004 when the ratio is followed by the small value in 2009 . Usually the ratio is significantly correlated with the days of sand-dust weather as the correlation coefficient is 0.683 (through the correlation test of significant level equal 0.01) It can be seen that the sand-dust weather has a certain effect on the average $\mathrm{PM}_{10}$ concentration. The effect is more obvious with sand and dusty weather occurrence increase.

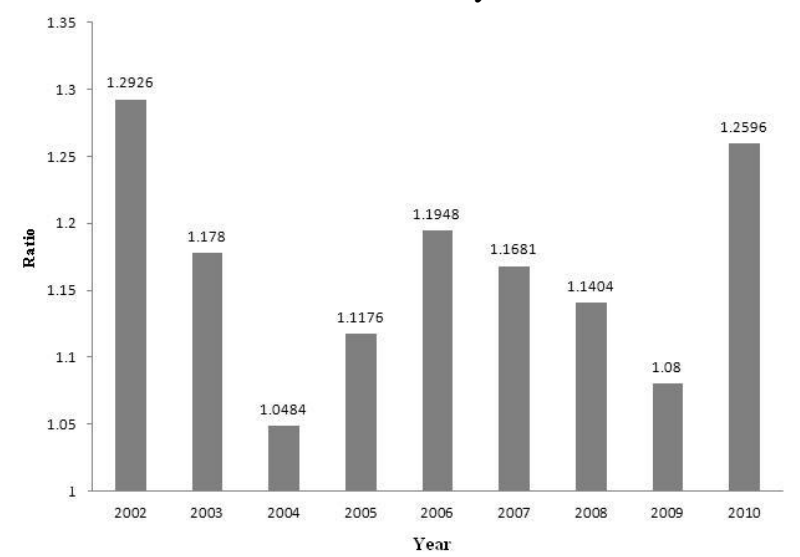

Fig. 3. Variation of the ratio between the average $\mathrm{PM}_{10}$ concentration and the average $\mathrm{PM}_{10}$ concentration that eliminates the value in the sand and dust weather.

\section{Characteristics of the Sand and Dust Weather}

Wind analysis finds that the most wind direction during the sand and dust weather is $0^{\circ}$ or $70^{\circ}$, respectively appears 31 times and 34 times. The Figure 4 presents that the main wind direction is located in the range from the north to the east in the spring in Lanzhou. Variable pressure in 3 hours is positive in the all years from 2003 to 2010 (Figure 5). The maximum reaches more than $30 \mathrm{hPa}$ in 2004. The variable pressure in positive is related to the weather system. A direct impact of the sand and dust weather is visibility. The average visibility eliminated sand-dust weather is $19 \mathrm{~km}$ in 2003 and 2004 (Figure 6). However, the seasonal average visibility obviously declines considering the occurrence of the sand and dust weather. The worst situation is that average visibility is less than a third of the visibility in eliminated sand-dust weather days. 


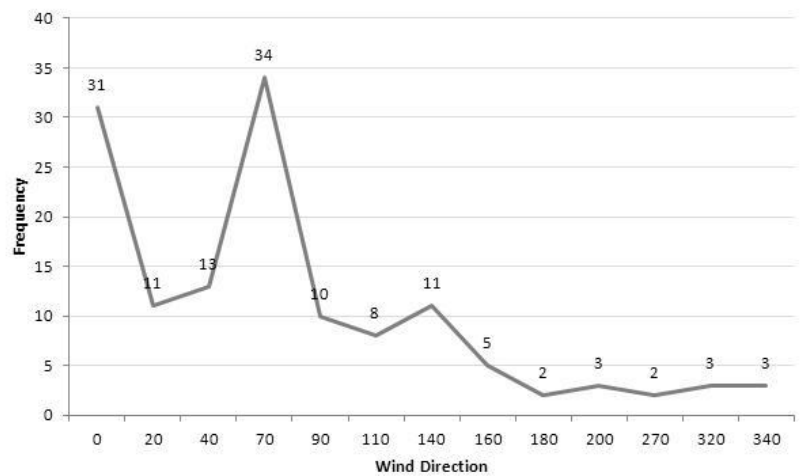

Fig. 4. Wind direction frequency in the sand and dust weather from 2001 to 2010.

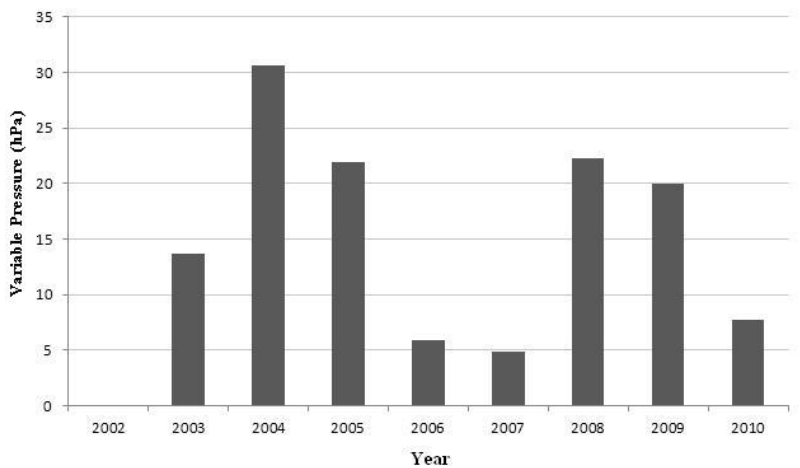

Fig. 5. Variation of the variable pressure in 3 hours during the sand and dust weather.

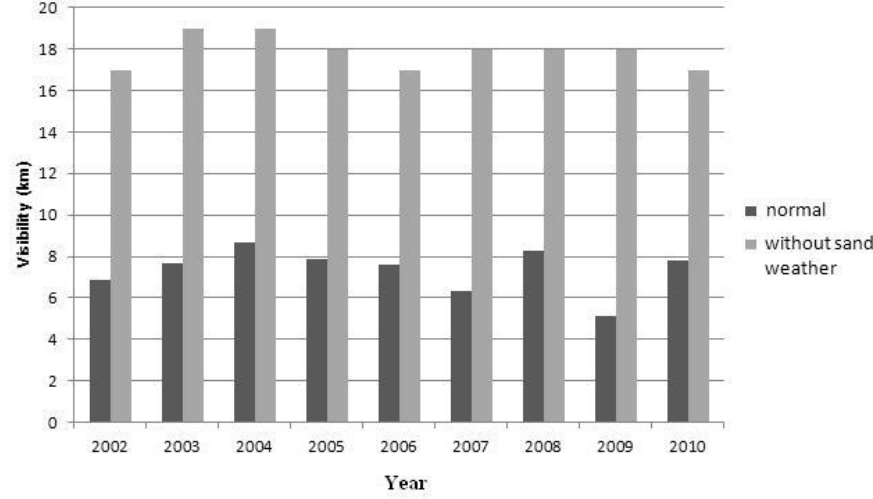

Fig. 6. Variation of the average visibility in the normal and visibility that sand and dust weather is eliminated in the spring from 2002 to 2010.

\section{Conclusions}

Impact of sand and dust weather on $\mathrm{PM}_{10}$ concentration is studied during 2001 to 2010 in Lanzhou. The conclusions are shown as following: 
(1)Spring is a season when sand-dust weather frequently occurs in Lanzhou. The occurrence of sand and dust weather shows a 4 years periodicity. The occurrence is obviously decreased after the peak.

(2)The daily average $\mathrm{PM}_{10}$ concentration is 1.16 times than the average $\mathrm{PM}_{10}$ concentration eliminated sand and dust weather. The occurrence of the sand and dust weather has improved $\mathrm{PM}_{10}$ average concentration. The effect is more obvious with days increase of the sand and dust weather.

(3) Variable pressure in 3 hours is positive in the process of sand-dust weather over Lanzhou. Wind direction mainly varies from the north to the east. Additional, sand and dust weather directly influences visibility. In the worst condition, average visibility is less than one third of the visibility if the sand and dust weather is eliminated in the spring.

\section{References}

1. Han Lanying, Zhang Qiang, Guo Ni, et al. Temporal and Spatial Characteristics of Dust Events in Northwest China[J]. Journal of Desert Research, 2012, 2: 025.

2. Yu Jianwei, Wang Yuan, Shen Shuqin, et al. Analysis on the Climate Change Characteristics of Sand/Dust Days as well as Its Temporal and Spatial Features over Jiangsu Region[J]. Scientia MeteorologicaSinica, 2008, 28(1): 45-49.

3. Wang Shigong, Yang Ming, Qi Bin, et al. Influence of Sand-dust Storms Occurring over the Gansu Hexi District on the Air Pollution in Lanzhou City[J]. Journal of Desert Research, 1999, 19(4): 354-358.

4. XueSishe, Gao Xueling, Yang Yuzhen, et al. Influence of Dust Events on Inspirable Particle Concentration in Cities of Shanxi Province[J]. Agriculture and Technology, 2012, 32(7): 117-119.

5. Yang Min, Wang Shigong, Li Wenli, et al. A Case Study of Sand-storm Effect on Environment of Lanzhou[J]. Meteorological Monthly, 2004, 4: 46-50.

6. Fan Yida, Shi Peijun, Zhou Tao, et al. The Impact Factor Analysis of Dust Storm Disaster in Northern China[J]. Advance in Earth Science, 2007, 22(4): 350-356. 